\title{
Effect of Poisson's Ratio on Large Amplitude Free Vibrations of Uniform Shear Flexible Beams
}

\author{
K.Meera Saheb ${ }^{\dot{A}} *$ and M.Shasikanth ${ }^{\dot{B}}$ \\ ${ }^{\grave{A}}$ Department of Mechanical Engineering University college of engineering(A), JNTU Kakinada-533 003, Andhra Pradesh, India \\ ${ }^{\dot{B}}$ Department of Mechanical Engineering MallaReddy College of Engineering, Hyderabad, Andhra Pradesh, India.
}

Accepted 10 January 2014, Available online 01 February 2014, Special Issue-2, (February 2014)

\begin{abstract}
Large amplitude vibration phenomenon of structural members like short (Timoshenko) beams requires the values of the Young's modulus and shear (rigidity) modulus. Though the shear modulus can be obtained from the Young's modulus, it is necessary to know the values of the Poisson ratio for the isotropic beams. The value of the Poisson ratio lies in a band for the isotropic materials. The generally used Poisson ratio for isotropic materials is in between 0.25 to 0.33 and most often is arbitrarily used in the analysis. The main aim of this paper is to study the effect of the variation of Poisson ratio with reference to the large amplitude free vibrations of beams, which is the simplest structural element. The coupled displacement field method is used in the analysis because of its inherent simplicity; where in the number of undetermined coefficients are reduced by a factor two in the admissible functions assumed for the lateral deflection and the total rotation. The present paper, though aimed at demonstrating the effect of Poisson ratio, on the large amplitude vibrations of Timoshenko beams, where in the effect of the transverse shear has to be considered, and a brief on method of analysis (coupled displacement formulation) is provided for the sake of completeness. For isotropic materials, the effect of transverse shear is linearly dependent on the shear modulus, which in turn is related to the Young's modulus through the Poisson ratio. The effect of taking a specific value of the Poisson ratio in the analysis is discussed in detail. The numerical results are obtained for several values of the Poisson ratio varying from 0 to 0.5 in steps of 0.1 . the numerical results show that the values of the Poisson ratio taken affects the ratios of the nonlinear to linear radian frequencies, for several amplitude ratios considered; the effect is seen to be considerable for higher amplitude and slenderness ratios.
\end{abstract}

Key words: Large amplitude vibrations, Poisson's ratio, coupled displacement field method.

\section{Introduction}

The classic work of Woinowsky - Kriegeron the large amplitude vibrations of slender beams inspired many a researcher to develop simple continuum formulations and finite element formulations with some simplifying assumptions. However, large amplitude vibrations of shear flexible beams have not been received much attention till recently except in the work of Rao et al., who have used a simplified finite element formulation.

Introduction of the effects of shear deformation and rotary inertia in the formulation yields coupled nonlinear differential equations. Further the oscillations being nonharmonic with two independent variables, namely, the lateral deflection and the total rotation, solution of such complicated equations by assuming both the spatial and temporal distribution is rather difficult. On the other hand, if the two variables appearing in the formulation are coupled to make the final equation a single nonlinear equation, then the solution becomes simpler. The reason

*Corresponding author: K.Meera Saheb being, in this system of single nonlinear equation, assuming suitable admissible functions in space eliminate the space variable and the resulting temporal equation can be solved using any standard method. This is the main advantage of the coupled displacement method. The authors have successfully applied this method to evaluate the large amplitude vibrations behavior of different structural members including beam. For the shear flexible beams, the value of the shear modulus is required. This value is evaluated from the Young's modulus which introduces the Poisson ratio. This ratio, for most of the isotropic metallic materials, varies between 0.25 to 0.33 and hence, a study on the variation of the Poisson ratio on the large amplitude vibrations of Timoshenko beams is necessary to find out the sensitivity of this ratio on the final results obtained in terms of the nonlinear to linear radian frequencies. Emphasis is given here in the fundamental mode of vibration as this requires minimum energy to get excited. In the next section the coupled displacement field method to obtain the ratio of nonlinear to the linear radian frequencies, which depends on the Poisson ratio used, for several maximum amplitude ratios. 


\section{Coupled Displacement Fields}

In this method, an admissible function for the lateral deflection $w$ that satisfies all the essential boundary conditions and symmetric condition, in the beam domain is assumed. The distribution for the total rotation $\theta$ is derived from the coupling equation. The $w$ distribution along the length of the beam is assumed as

$w=a \sin \frac{\pi x}{L}$

where ' $a$ ' is the central lateral deflection of the beam.

The coupling equation is taken, as

$\frac{d w}{d x}=-\theta+\frac{E I}{k G A} \theta^{\prime}$

Substituting the function $w$ in equation (2), the displacement field for ' $\theta$ ', is obtained after solving the above differential equation, as

$\theta=\lambda \operatorname{acos} \frac{\pi x}{L}$

where

$$
\lambda=\frac{-\frac{\pi}{L}}{\left\{1+\left(\frac{\pi}{L}\right)^{2} \frac{E I}{k G A}\right\}}
$$

It may be noted here that because of the coupled field method ' $\theta$, the total rotation field distribution, contains the same undetermined coefficient ' $a$ ' as in the $w$ distribution.

\section{Large Amplitude Vibrations - Energy Method}

Large amplitude vibrations can be studied, once the coupled displacement field for the total rotation ' $\theta$ ', for an assumed ' $w$ ' distribution is evaluated using the principle of conservation of total energy at any instant of time, which states that

\section{$\mathrm{U}+\mathrm{T}+\mathrm{W}=$ Constant}

The expression for $\mathrm{U}, \mathrm{T}$ and $\mathrm{W}$ are given by

$U=\frac{E I}{2} \int_{o}^{L}\left(\frac{d \theta}{d x}\right)^{2} d x+\frac{k G A}{2} \int_{o}^{L}\left(\frac{d w}{d x}+\theta\right)^{2} d x$

$T=\frac{\rho A}{2} \int_{0}^{L} \dot{w}^{2} d x+\frac{\rho I}{2} \int_{0}^{L} \dot{\theta}^{2} d x$

$W=\frac{T_{a}}{2} \int_{o}^{L} \frac{1}{2}\left(\frac{d w}{d x}\right)^{2} d x$

where $U$ is the strain energy, $T$ is the kinetic energy, $W$ is the work done by the tension developed because of large amplitudes (deflections), ' $\rho$ ' is the mass density, ' $A$ ' is the area of cross - section, ' $I$ ' is the area moment of inertia, $T_{a}$ is the tension developed in the beam because of large deflections, and $\left({ }^{\circ}\right)$ denotes differentiation with respect to time.

$\mathrm{T}_{\mathrm{a}}$ is evaluated, following Woinowsky - Krieger, as

$T_{a}=\frac{E I}{2 L r^{2}} \int_{o}^{L}\left(\frac{d w}{d x}\right)^{2} d x$

where ' $r$ ' is the radius of gyration.

It is to be noted here that ' $w$ ' in Eq. (9) does not contain shear flexible terms, and $T_{a}$ is evaluated in terms of nondimensional amplitude $q(=a / r)$.

Substituting the expressions for $w$ and $\theta$ (obtained from the coupling equation), the expressions for $U, T$ and $W$ are evaluated and after simplification the temporal equation in terms of the nondimensional amplitude is obtained, as

$$
\dot{q}^{2}+\alpha_{1} q^{2}+\alpha_{2} q^{4}=\text { constant }
$$

where

$$
\alpha_{l}=\frac{E I\left(\frac{\pi}{L}\right)^{4}\left[1+\frac{\pi^{2} E}{k G \beta^{2}}\right]}{\rho A\left\{\left[1+\frac{\pi^{2} E}{k G \beta^{2}}\right]^{2}+\frac{\pi^{2}}{\beta^{2}}\right\}}
$$

$\alpha_{2}=\frac{E I\left(\frac{\pi}{L}\right)^{4}\left[1+\frac{\pi^{2} E}{k G \beta^{2}}\right]^{2}}{8 \rho A\left\{\left[1+\frac{\pi^{2} E}{k G \beta^{2}}\right]^{2}+\frac{\pi^{2}}{\beta^{2}}\right\}}$

In Eqs. (11) and (12) $G$ can be expressed in terms of $E$ from the standard relation,

$$
G=\frac{E}{2(1+v)}
$$

where $v$ is the Poisson ratio and $\beta(=\mathrm{L} / \mathrm{r})$ is the slenderness ratio of the beam.

From Eq. (10), following the procedure given in Ref., and applying the harmonic balance method, the ratios of the nonlinear to the linear radian frequencies can be obtained, as

$$
\begin{aligned}
& \left(\frac{\omega_{N L}}{\omega_{L}}\right)^{2}=1+\frac{3}{16}\left[1+\frac{\pi^{2} E}{k G \beta^{2}}\right]\left(\frac{a_{m}}{r}\right)^{2} \\
& \left(\frac{\omega_{N L}}{\omega_{L}}\right)^{2}=1+\frac{3}{16}\left[1+\frac{23.6871(1+v)}{\beta^{2}}\right]\left(\frac{a_{m}}{r}\right)^{2}
\end{aligned}
$$

For very large $\beta$, i.e., for slender beams, where the shear deformation and rotary inertia can be neglected, Eq. (14) becomes

$\left(\frac{\omega_{N L}}{\omega_{L}}\right)^{2}=1+\frac{3}{16}\left(\frac{a_{m}}{r}\right)^{2}$

which is a standard result in the case of a clampedclamped beam, a single term trigonometric admissible function for $\theta$ that satisfies the required boundary conditions is taken as

$$
w=a\left[1-\frac{\cos 2 \pi x}{L}\right]
$$


Table1 $\omega_{\mathrm{NL}} / \omega_{\mathrm{L}}$ Values for the Poisson's ratio of 0.3 for Hinged-Hinged beam

\begin{tabular}{|c|c|c|c|c|c|c|c|c|c|c|}
\hline \multirow{4}{*}{$\mathrm{a}_{\mathrm{m}} / \mathrm{r}$} & \multicolumn{10}{|c|}{$v=0.3$} \\
\hline & \multicolumn{10}{|c|}{$\beta$} \\
\hline & \multicolumn{3}{|c|}{25} & \multicolumn{3}{|c|}{50} & \multicolumn{3}{|c|}{100} & $\rightarrow \propto$ \\
\hline & Present Study & $\begin{array}{c}\text { FEM } \\
{[7]}\end{array}$ & $\begin{array}{c}\text { DNI } \\
{[3]}\end{array}$ & $\begin{array}{l}\text { Present } \\
\text { Study }\end{array}$ & $\begin{array}{c}\text { FEM } \\
\text { [7] }\end{array}$ & $\begin{array}{c}\text { DNI } \\
{[3]}\end{array}$ & $\begin{array}{c}\text { Present } \\
\text { Study }\end{array}$ & $\begin{array}{c}\text { FEM } \\
{[7]}\end{array}$ & $\begin{array}{c}\text { DNI } \\
{[3]}\end{array}$ & \\
\hline 0.0 & 1.0000 & 1.0000 & 1.0000 & 1.0000 & 1.0000 & 1.0000 & 1.0000 & 1.0000 & 1.0000 & 1.0000 \\
\hline 0.2 & 1.0039 & - & 1.0039 & 1.0038 & - & 1.0038 & 1.0038 & - & 1.0038 & 1.0037 \\
\hline 0.4 & 1.0156 & 1.0156 & 1.0156 & 1.0151 & 1.0150 & 1.0151 & 1.0149 & 1.0149 & 1.0149 & 1.0149 \\
\hline 0.6 & 1.0348 & - & 1.0347 & 1.0336 & - & 1.0335 & 1.0333 & - & 1.0332 & 1.0332 \\
\hline 0.8 & 1.0611 & 1.0605 & 1.0608 & 1.0590 & 1.0585 & 1.0588 & 1.0585 & 1.0585 & 1.0582 & 1.0583 \\
\hline 1.0 & 1.0940 & 1.0925 & 1.0933 & 1.0905 & 1.0897 & 1.0902 & 1.0900 & 1.089 & 1.8940 & 1.0897 \\
\hline 2.0 & 1.3368 & 1.3213 & 1.3313 & 1.3264 & 1.3142 & 1.3212 & 1.3237 & 1.3125 & 1.3186 & 1.3229 \\
\hline 3.0 & 1.6645 & 1.6146 & 1.6501 & 1.6457 & 1.6052 & 1.6318 & 1.6409 & 1.6257 & 1.6272 & 1.6394 \\
\hline 4.0 & 2.0366 & - & 2.0116 & 2.0092 & - & 1.9850 & 2.0023 & - & 1.9783 & 2.0000 \\
\hline 5.0 & 2.4328 & - & 2.3968 & 2.3969 & - & 2.3619 & 2.3879 & - & 2.3531 & 2.3848 \\
\hline
\end{tabular}

Table $2 \omega_{\mathrm{NL}} / \omega_{\mathrm{L}}$ Values for the Values of Poisson's ratios of 0.4 and 0.5 for Hinged-Hinged beam

\begin{tabular}{|c|c|c|c|c|c|c|c|c|}
\hline \multirow{4}{*}{$\frac{a_{m}}{r}$} & \multicolumn{8}{|c|}{$v$} \\
\hline & \multicolumn{4}{|c|}{0.4} & \multicolumn{4}{|c|}{0.5} \\
\hline & \multicolumn{4}{|c|}{$\beta$} & \multicolumn{4}{|c|}{$\beta$} \\
\hline & 25 & 50 & 100 & $\rightarrow \propto$ & 25 & 50 & 100 & $\rightarrow \propto$ \\
\hline 0.0 & 1 & 1 & 1 & 1 & 1 & 1 & 1 & 1 \\
\hline 0.2 & 1.0039 & 1.0038 & 1.0038 & 1.0037 & 1.0040 & 1.0038 & 1.0038 & 1.0037 \\
\hline 0.4 & 1.0157 & 1.0151 & 1.0149 & 1.0149 & 1.0157 & 1.0151 & 1.0149 & 1.0149 \\
\hline 0.6 & 1.0349 & 1.0336 & 1.0333 & 1.0332 & 1.0351 & 1.0337 & 1.0333 & 1.0332 \\
\hline 0.8 & 1.0613 & 1.0591 & 1.0585 & 1.0583 & 1.0615 & 1.0591 & 1.0585 & 1.0583 \\
\hline 1.0 & 1.0943 & 1.0909 & 1.0900 & 1.0897 & 1.0946 & 1.0910 & 1.0900 & 1.0897 \\
\hline 2.0 & 1.3378 & 1.3266 & 1.3238 & 1.3229 & 1.3389 & 1.3269 & 1.3239 & 1.3229 \\
\hline 3.0 & 1.6664 & 1.6462 & 1.6411 & 1.6394 & 1.6684 & 1.6467 & 1.6412 & 1.6394 \\
\hline 4.0 & 2.0394 & 2.0099 & 2.0025 & 2.0000 & 2.0422 & 2.0106 & 2.0027 & 2.0000 \\
\hline 5.0 & 2.4364 & 2.3979 & 2.3881 & 2.3849 & 2.4401 & 2.3988 & 2.3883 & 2.3849 \\
\hline
\end{tabular}

and by following the similar procedure as discussed for hinged-hinged beam the ratios of the nonlinear to the linear radian frequencies can be obtained for the clampedclamped beam, as

$\left(\frac{\omega_{N L}}{\omega_{L}}\right)^{2}=1+\frac{3}{64}\left[1+\frac{94.7482(1+v)}{\beta^{2}}\right]\left(\frac{a_{m}}{r}\right)^{2}$

\section{Numerical Results and Discussion}

Using the coupled displacement formulation, the large amplitude behaviour of a uniform shear flexible hinged beam is obtained in terms of $\omega_{\mathrm{NL}} / \omega_{\mathrm{L}}$ (ratios of nonlinear to the linear radian frequency) for various $a_{m} / r$ (maximum amplitude ratios) , L/r (slenderness ratios) and
Poisson ratio $(v)$. The beam considered has axially immovable ends (Fig.1).

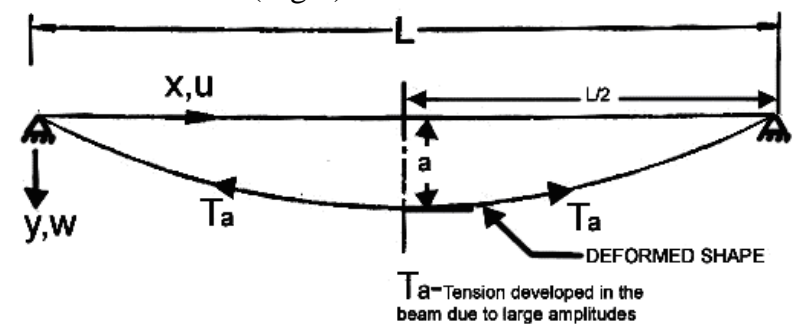

Fig. 1. A Shear Flexible Hinged- Hinged Beam Undergoing Large Deflections

The present results in terms of the ratios of the nonlinear to the linear radian frequencies are presented in Table 1 
Table3 $\omega_{\mathrm{NL}} / \omega_{\mathrm{L}}$ Values for the Poisson's ratio of 0.3 for Clamped-Clamped beam

\begin{tabular}{|c|c|c|c|c|c|c|c|c|c|c|}
\hline \multirow{4}{*}{$\mathrm{a}_{\mathrm{m}} / \mathrm{r}$} & \multicolumn{10}{|c|}{$v=0.3$} \\
\hline & \multicolumn{10}{|c|}{$\beta$} \\
\hline & \multicolumn{3}{|c|}{25} & \multicolumn{3}{|c|}{50} & \multicolumn{3}{|c|}{100} & $\rightarrow \propto$ \\
\hline & Present Study & $\begin{array}{c}\text { FEM } \\
{[7]} \\
\end{array}$ & $\begin{array}{c}\text { DNI } \\
{[3]} \\
\end{array}$ & $\begin{array}{c}\text { Present } \\
\text { Study }\end{array}$ & $\begin{array}{c}\text { FEM } \\
{[7]} \\
\end{array}$ & $\begin{array}{c}\text { DNI } \\
{[3]}\end{array}$ & $\begin{array}{l}\text { Present } \\
\text { Study }\end{array}$ & $\begin{array}{c}\text { FEM } \\
{[7]}\end{array}$ & $\begin{array}{c}\text { DNI } \\
{[3]}\end{array}$ & \\
\hline 0.0 & 1.0000 & 1.0000 & 1.0000 & 1.0000 & 1.0000 & 1.0000 & 1.0000 & 1.0000 & 1.0000 & 1.0000 \\
\hline 0.2 & 1.0011 & - & 1.0011 & 1.0010 & - & 1.0010 & 1.0009 & - & 1.0009 & 1.0009 \\
\hline 0.4 & 1.0045 & 1.0390 & 1.0045 & 1.0039 & 1.0036 & 1.0039 & 1.0038 & 1.0035 & 1.0038 & 1.0037 \\
\hline 0.6 & 1.0100 & - & 1.0100 & 1.0088 & - & 1.0088 & 1.0085 & - & 1.0085 & 1.0084 \\
\hline 0.8 & 1.0178 & 1.0153 & 1.0178 & 1.0156 & 1.0142 & 1.0156 & 1.0151 & 1.0140 & 1.0151 & 1.0149 \\
\hline 1.0 & 1.0277 & 1.0238 & 1.0276 & 1.0243 & 1.0228 & 1.0242 & 1.0235 & 1.0218 & 1.0234 & 1.0232 \\
\hline 2.0 & 1.1065 & 1.0895 & 1.1058 & 1.0940 & 1.0800 & 1.0933 & 1.0908 & 1.0834 & 1.0902 & 1.0897 \\
\hline 3.0 & 1.2268 & 1.1855 & 1.2239 & 1.2011 & 1.1771 & 1.1987 & 1.1946 & 1.1759 & 1.1924 & 1.1924 \\
\hline 4.0 & 1.3776 & - & 1.3711 & 1.3368 & - & 1.3313 & 1.3264 & - & 1.3212 & 1.3229 \\
\hline 5.0 & 1.5501 & - & 1.5389 & 1.4932 & - & 1.4836 & 1.4786 & - & 1.4694 & 1.4737 \\
\hline
\end{tabular}

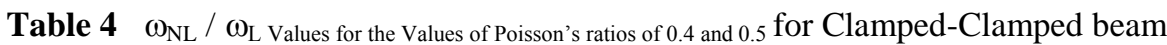

\begin{tabular}{|c|c|c|c|c|c|c|c|c|}
\hline$a$ & \multicolumn{8}{|c|}{$v$} \\
\hline \multirow{3}{*}{$\frac{m}{r}$} & \multicolumn{4}{|c|}{0.4} & \multicolumn{4}{|c|}{0.5} \\
\hline & \multicolumn{4}{|c|}{$\beta$} & \multicolumn{4}{|c|}{$\beta$} \\
\hline & 25 & 50 & 100 & $\rightarrow \propto$ & 25 & 50 & 100 & $\rightarrow \propto$ \\
\hline 0.0 & 1 & 1 & 1 & 1 & 1 & 1 & 1 & 1 \\
\hline 0.2 & 1.0011 & 1.0010 & 1.0010 & 1.0010 & 1.0011 & 1.0010 & 1.0010 & 1.0010 \\
\hline 0.4 & 1.0045 & 1.0039 & 1.0038 & 1.0037 & 1.0046 & 1.0039 & 1.0038 & 1.0037 \\
\hline 0.6 & 1.0102 & 1.0089 & 1.0085 & 1.0084 & 1.0103 & 1.0089 & 1.0085 & 1.0084 \\
\hline 0.8 & 1.0180 & 1.0157 & 1.0151 & 1.0149 & 1.0182 & 1.0157 & 1.0151 & 1.0149 \\
\hline 1.0 & 1.0280 & 1.0244 & 1.0235 & 1.0232 & 1.0284 & 1.0245 & 1.0235 & 1.0232 \\
\hline 2.0 & 1.1078 & 1.0944 & 1.0909 & 1.0898 & 1.1091 & 1.0945 & 1.0910 & 1.0898 \\
\hline 3.0 & 1.2293 & 1.2019 & 1.1948 & 1.1925 & 1.2319 & 1.2023 & 1.1950 & 1.1925 \\
\hline 4.0 & 1.3809 & 1.3381 & 1.3267 & 1.3230 & 1.3856 & 1.3387 & 1.3269 & 1.3230 \\
\hline 5.0 & 1.5556 & 1.4950 & 1.4790 & 1.4739 & 1.5613 & 1.4958 & 1.4794 & 1.4739 \\
\hline
\end{tabular}

and Table 3 for the Poisson's ratio of 0.3 . For the sake of comparison and validation of the proposed method, the same results obtained by the finite element method (FEM) and by using the direct numerical integration method (DNI) are included in the same Table 1 and Table 3 for the commonly used Poisson's ratio of 0.3. The present results in terms of $\omega_{\mathrm{NL}} / \omega_{\mathrm{L}}$ are presented in Table 2 and Table 4 for the value of the Poisson's ratio of 0.4 and 0.5 .

From the results presented to investigate the effect of the Poisson's ratio on the nonlinear vibrations of isotopic, uniform, shear flexible and homogeneous beams with axially immovable ends the following broad observations can be made:

- The effect of the Poisson's ratio is negligible for lower amplitude ratios and is considerable for higher amplitude ratios of the vibrating beam.

- The effect of the Poisson's ratio for high slenderness ratios of the beam is negligible and for the smaller slenderness ratios is considerable.
- The effect of the increasing Poisson's ratio, from 0.3 to 0.5 , on the nonlinear vibrations is considerable.

- Nonlinearities are more predominant in case of hinged-hinged beam when compared to clamped clamped beam at higher amplitude ratios.

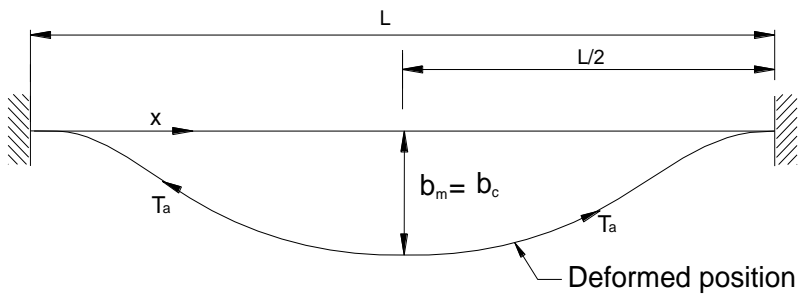

Fig. 2. A shear flexible clamped-Clamped beam undergoing large deflections. 


\section{Conclusions}

The effect of the variation of the Poisson's ratio, with respect to the nonlinear (large amplitude) vibrations is studied using the coupled displacement field formulation of a uniform shear flexible hinged beams, with the ends of the beam are taken as axially immovable. This classical problem is chosen as a demonstration problem, to show the effect of variation of the Poisson's ratio on the nonlinear vibrations. Though the theoretical bounds of the Poisson's ratio are -1 and 0.5 , the variation of the Poisson's ratio considered in the present study is 0.0 to 0.5 , as a negative Poisson's ratio is not applicable for isotropic shear flexible beams.

\section{Acknowledgements}

The author is thankful to the authorities of University College of Engineering(A), JNTU-Kakinada for sponsoring the necessary economical support for presenting the paper under TEQIP phase2. Second author gratefully acknowledges the support given by Mallareddy college of engineering during the course of this work.

\section{References}

S. Woinowsky - Krieger, (1950) The effect of an axial force on the vibration of hinged bars, Journal of Applied Mechanics, 17, pp. 35-36.

A.V. Srinivasan, (1956) Large amplitude free oscillations of beams and plates, AIAA Journal3 pp.1951-1953.

G.V. Rao, and K.K. Raju, (2002) A direct numerical integration method to study the large amplitude vibration of slender beams with immovable ends, Journal of Institute of Engineers (I) pp. 42-44.

G.V.Rao and K.K. Raju, (2003) Large amplitude free vibrations of beams - an energy approach, ZAMM 8 pp.493-498.

C. Mei, (1972) Non-linear vibrations of beams by Matrix displacement method, AIAA Journal10 pp.355-357.

G.V.Rao G.V,I.S. Raju, and K.K.Raju, (1976) Finite element formulation for the large amplitude free vibrations of beams and orthotropic circular plates, Computers \& Structures, 6, pp.169-172.

G.V.Rao, I.S.Raju and K.K.Raju, (1976) Non-linear vibrations of beams considering shear deformation and rotary inertia, AIAA Journal14, pp.685-687.

G.V. Rao, K.Meera Saheb and G. Rangajanardhan, (2006) Fundamental frequency for large amplitude vibrations of uniform Timoshenko beams with central point concentrated mass using coupled displacement field method, Journal of Sound and Vibration, 298, pp. 221-232.
G.V. Rao, K.Meera Saheb and G. Rangajanardhan, (2006) Concept of coupled displacement filed for large amplitude free Vibrations of shear flexible beams, Journal of Vibration and Acoustics (ASME), 128. pp.251-255.

K.Meera Saheb, G.V. Rao and G.Ranga Janardhana,(2007) Coupled Displacement Field formulation for the Buckling analysis of shear flexible columns, Journal of Structural Engineering, 33, pp.413-418.

K.Meera Saheb G.V.Rao and G.Ranga Janardhana,(2007) Free Vibration Analysis of Timoshenko Beams using coupled Displacement Field Method, Journal of structural Engineering, 34, pp.233-236.

K.Meera Saheb G.V. Rao and G.Ranga Janardhana, (2009) Buckling and free vibration of moderately thick square plates using coupled displacement field method, Journal of structural Engineering, 36, pp.155-159.

G.V.Rao and K.Meera Saheb, (2004)Large amplitude free vibrations of shear flexible uniform beams through coupled displacement field, A FUSION OF HARMONICS, proceedings of the 3rd International Conference on Vibration Engineering and Technology of Machinery (VETOMAC-3) and the 4th-Pacific Conference on system Integrity and Maintenance (ACSIM-2004), edited by Vyas et al, Organized by the IIT, Kanpur, India and the cooperative Research Center on the Integrated Engineering and Asset Management (CIEAM), Australia, Allied Publishers, private limited, NEW DELHI, Vol, II, pp. 1167-1174(Also paper presented at the conference, New Delhi, 6-9 December, 2004).

K.Meera Saheb, G.V. Rao and G.Rangajanardhan, (2007) Evaluation Of Large Amplitude Free Vibration Behavior Of Uniform Timoshenko Beams Using Coupled Displacement Field Method ,Proceedings International conference on Vibration Engineering \& Technology of Machinery(VETOMAC-4),Organisied by university college of Engineering, Osmania University, India \& corporate R \& D Division, BHEL India, in collaboration with the vibration institute of India,pp.340-348 (Also paper presented at the conference, Hyderabad, 17-19 December, 2007).

K.Meera Saheb, C.Sashikant and G.V. Rao, (2013) Evaluation of Large Amplitude Free Vibration Behavior of Moderately Thick Square Plates using Coupled Displacement Field Method, Proceedings of International conference on Vibration Engineering \& Technology of Machinery(VETOMAC-9), Organized by Nanjing university of Aeronautics and Astronautics, Nanjing, P.R.China (Also paper presented at the conference, Nanjing, P.R. China, 20-23 August, 2013). 